\title{
EFEITO HORMÓTICO DE GLIPHOSATE NO DESENVOLVIMENTO INICIAL DE CANA-DE-AÇÚCAR ( $\left.{ }^{1}\right)$
}

\author{
MARCELO DE ALMEIDA SILVA $\left({ }^{2 *}\right)$; NATÁLIA CAROLINA ARAGÃO $\left({ }^{3}\right)$; MARIANA DE ALMEIDA \\ BARBOSA $\left({ }^{4}\right)$; ELISANGELA MARQUES JERONIMO $\left({ }^{2}\right)$; SAMIRA DOMINGUES CARLIN $\left({ }^{2}\right)$
}

\begin{abstract}
RESUMO
Efeito hormótico é definido como o efeito estimulante de pequenas doses de substâncias, as quais em doses maiores são inibitórias. Esta pesquisa objetivou verificar, em casa-de-vegetação, o efeito de subdoses do herbicida gliphosate no desenvolvimento inicial de cana-de-açúcar. Plantas de cana-de-açúcar foram obtidas de gemas isoladas plantadas em vasos plásticos de 2,5 L. Aos 50 dias após o plantio, gliphosate foi aplicado nas doses de $0 ; 1,8 ; 3,6 ; 7,2 ; 18 ; 36 ; 72 ; 180 ; 360$ e 720 g e.a. ha ${ }^{-1}$. Aos 0 e 25 dias após a aplicação (DAA) foram avaliados altura da planta, número de perfilhos, número de folhas verdes, número de folhas secas e estimativa do conteúdo de clorofila (índice SPAD). Aos 25 DAA também foram determinadas a massa fresca e seca da parte aérea e raízes das plantas. O delineamento experimental utilizado foi o inteiramente casualizado com quatro repetições. A dose de $1,8 \mathrm{~g}$ e.a. ha ${ }^{-1}$ de gliphosate estimulou as características de crescimento no desenvolvimento inicial da cana-de-açúcar. Esse efeito hormótico poderá ser utilizado como manejo da cultura para obter melhor exploração do ambiente de produção.
\end{abstract}

Palavras-chave: Saccharum spp., herbicida, subdose, estímulo no crescimento.

\section{ABSTRACT \\ HORMETIC EFFECT OF GLYPHOSATE ON THE INITIAL DEVELOPMENT OF SUGARCANE}

Hormesis is defined as the stimulating effect of small doses of substances, which in larger doses are inhibitory. This research aimed at evaluating the effect of low doses of the herbicide glyphosate on the initial development of sugarcane in greenhouse. Sugarcane plants were obtained from isolated buds planted in plastic pots of $2.5 \mathrm{~L}$. At 50 days after planting, glyphosate was sprayed at $0,1.8,3.6,7.2,18,36$, $72,180,360$ and $720 \mathrm{~g} \mathrm{AE} \mathrm{ha}^{-1}$. At 0 and 25 days after application (daa), plant height, tiller number, number of green leaves, number of dead leaves and estimated chlorophyll contents (index SPAD) were evaluated. At 25 evaluated daa, fresh and dry mass of above-ground plant and roots were also determined. The experiment was installed in a completely randomized design with four replications. The glyphosate dose of $1.8 \mathrm{~g} \mathrm{AE} \mathrm{ha}^{-1}$ stimulated growth traits at the initial development of sugarcane. This hormetic effect could be used to manage the crop to exploit better the production environment.

Key words: Saccharum spp., herbicide, low dose, growth stimulus.

$\left({ }^{1}\right)$ Recebido para publicação em 3 de junho de 2008 e aceito em 28 de maio de 2009.

$\left({ }^{2}\right)$ Polo Centro-Oeste - APTA, Caixa Postal 66, 17201-970 Jaú (SP), Brasil. E-mail: marcelosilva@apta.sp.gov.br (*) Autor correspondente.

$\left({ }^{3}\right)$ Graduanda de Biologia da Universidade Sagrado Coração/USC, Bauru (SP), Brasil. Bolsista FUNDAP.

$\left({ }^{4}\right)$ Graduanda de Biologia da Universidade Estadual Paulista/UNESP, Botucatu (SP), Brasil. 


\section{INTRODUÇÃO}

Os reguladores vegetais têm demonstrado cada vez mais importância na agricultura tropical, à medida que as técnicas de cultivo evoluem, principalmente, em culturas de alto valor econômico. São produtos sintéticos que possuem as mesmas características que os hormônios vegetais, ou seja, atuam promovendo, inibindo ou alterando os processos bioquímicos e fisiológicos nas diferentes estruturas celulares das plantas. Os que estimulam o crescimento estão relacionados à divisão, alongamento e diferenciação celular, enquanto aqueles que inibem o crescimento referem-se à senescência, amadurecimento de frutos e dormência de sementes (CAstro e Vieira, 2001).

Existem produtos químicos, originalmente utilizados como herbicidas, que em subdoses auxiliam no desenvolvimento da planta. Southam e ErLich (1943) relataram que um composto originado a partir da casca de carvalho, submetido em subdosagens, estimulou o desenvolvimento de fungos, e que, em doses elevadas foi tóxico e inibiu o crescimento.

Atualmente, tanto no setor de produção agrícola quanto nas diversas áreas da saúde humana, o efeito de subdoses de produtos aplicados, denominado como hormótico, vem sendo amplamente discutido e pesquisado, com o objetivo de compreender o mecanismo de ação estimulante e benéfica de diversas substâncias inicialmente consideradas tóxicas.

Resultados referentes ao efeito hormótico foram verificados por meio de aplicações do herbicida simazine, o qual proporcionou aumento protéico em plantas de aveia (Pulver e Ries, 1973) e centeio (Ries et al., 1967). Assim como o oxyfluorfen ocasionou maior resistência a patógenos na cultura da soja (Nelson et al., 2002) e os herbicidas dalapon, bromoxynll e terbacil estimularam o crescimento em plantas de trigo (Wiedman e ApPLeby, 1972).

O gliphosate (N-phosphonometyl-glycina) é um composto usado em ampla escala na agricultura. Trata-se de um herbicida de ação sistêmica, largo espectro, não seletivo, com translocação via simplasto (Franz, 1985). O gliphosate inibe a enzima EPSPs (sintetase fosfato do ácido enolpiruvato chiquímico), impedindo a formação do metabolismo secundário. Simultâneamente a atividade da enzima PAL (fenilalanina amonioliase) é estimulada, promovendo elevação da síntese de compostos fenólicos. Além disso, ocorre significativa diminuição da síntese proteica e elevação de compostos tóxicos, estimulando a produção de etileno, levando a planta à degeneração celular.
Pesquisas foram realizadas com subdoses de gliphosate no desenvolvimento inicial de plantas de trapoeraba (Commelina benghalensis) (MEschede et al., 2007a), Eucaliptus grandis (CARBONARI et al., 2007b) e citrus (CARBONARI et al., 2007a). Estes mesmos autores concluíram que há efeito positivo no crescimento, aumento da biomassa, desenvolvimento da parte aérea e do sistema radicular das plantas estudadas, bem como incrementos na absorção e acúmulo de fósforo nas folhas.

Entretanto, segundo resultados observados por MACIEL et al. (2007) com plantas de curauá branco (Ananas erectifolius), a aplicação de subdoses de gliphosate não provocou estímulo ou incremento no desenvolvimento vegetativo.

$\mathrm{Na}$ cultura da cana-de-açúcar (Saccharum spp.), o gliphosate é amplamente empregado como herbicida no controle de plantas daninhas, em doses que variam de 64,8 a 777,6 g e.a. ha ${ }^{-1}$ (BLANCO, 2003), além de ser utilizado como maturador, nas doses de 108,0 a 216,0 g e.a. ha ${ }^{-1}$ (NetTo, 2006) e também na eliminação de soqueiras para fins de renovação da cultura, com doses de 1800 a 2160 g e.a. ha ${ }^{-1}$ (SILVA et al., 2006).

O objetivo deste estudo foi avaliar a resposta de plantas de cana-de-açúcar em estágio inicial de desenvolvimento à aplicação de subdoses do herbicida gliphosate.

\section{MATERIAL E MÉTODOS}

O experimento foi desenvolvido em casa de vegetação em Jaú (SP). Foram utilizados vasos plásticos com 2,5 litros de substrato Plantmax ${ }^{\circledR}$ (Eucatex Agro). A cada 100 litros de substrato foram misturadas $1200 \mathrm{~g}$ do fertilizante formulado 08-28-16. Gemas individualizadas da variedade SP80-1842 foram plantadas em cada vaso.

Utilizou-se o delineamento inteiramente casualizado, em esquema fatorial $10 \times 2$, constituído por 10 doses de gliphosate $(0 ; 1,8 ; 3,6 ; 7,2 ; 18 ; 36 ; 72$; 180; 360 e 720 g e.a. ha ${ }^{-1}$ ) e duas épocas de avaliação (0 e 25 dias após a aplicação-daa), com quatro repetições.

A aplicação do gliphosate foi realizada aos 50 dias após o plantio das gemas por meio de um pulverizador costal manual, com barra de pulverização munida de um bico de latão tipo Magnum defletor 0,50, volume de calda de $200 \mathrm{~L} \mathrm{ha}^{-1}$ e pressão de aplicação de 2 bar. Antes da pulverização (época 0 daa) e aos 25 daa foram avaliadas as seguintes variáveis: altura da planta (A), medida por meio de fita métrica da base até o primeiro dewlap 
visível (folha +1$)$ do perfilho primário; número de perfilhos (NP); número de folhas verdes (FV); número de folhas secas (FS) e estimativa do conteúdo de clorofila, por meio do índice SPAD, obtido com clorofilômetro portátil SPAD-502 (Minolta Corp., EUA), média de quatro leituras tomadas na folha +1 .

No fim do experimento efetuou-se a colheita, separando-se manualmente a parte aérea do sistema radicular das plantas de todos os vasos. Todo o substrato foi peneirado, separando-se o sistema radicular e, a seguir, lavado. Quantificou-se a massa úmida de todo o material vegetal: ponteiros com folhas verdes e secas, e sistema radicular. A massa seca desse material foi obtida após secagem em estufa de ventilação forçada a $65^{\circ} \mathrm{C}$ por 72 horas.

Os resultados foram submetidos à análise de variância, utilizando o teste $\mathrm{F}$ a $95 \%$.
Posteriormente, para as causas de variação significativas, foi aplicado o teste de Scott-Knott ( $\mathrm{p}$ $<0,05)$ para agrupamento das médias.

\section{RESULTADOS E DISCUSSÃO}

A análise da variância indicou significância das causas de variação doses (D) para número de perfilhos, altura da planta, folhas verdes e índice SPAD. Quanto à época de avaliação (E), foi observado efeito significativo na altura da planta, folhas verdes, folhas secas e índice SPAD (Tabela 1). Para as interações (Tabela 1), verificouse significância no caso da altura de plantas, folhas verdes e índice SPAD. Tais interações significativas são indicativas da interdependência dos fatores em estudo.

Tabela 1. Valores de quadrado médio da análise de variância para números de perfilhos (NP), altura da planta (A), folhas verdes (FV), folhas secas (FS) e estimativa do conteúdo de clorofila (índice SPAD) com aplicação de doses de gliphosate

\begin{tabular}{lccccc}
\hline & NP & A & FV & FS & SPAD \\
\hline Doses (D) & $26388,9^{*}$ & $1043319,3^{* *}$ & $451111,1^{* *}$ & $46833,3^{\text {ns }}$ & $641429,8^{* *}$ \\
Época (E) & $166,7^{\text {ns }}$ & $8169660,0^{* *}$ & $1472666,7^{* *}$ & $4988166,7^{*}$ & $38064735,0^{* *}$ \\
DxE & $10537,0^{\text {ns }}$ & $892989,6^{* *}$ & $173037,1^{*}$ & $23722,2^{\text {ns }}$ & $617883,1^{* *}$ \\
\hline CV (\%) & 26,67 & 11,06 & 30,63 & 53,80 & 3,44 \\
\hline Média Geral & 3,75 & 28,83 & 9,17 & 3,82 & 39,67 \\
\hline
\end{tabular}

Teste de Scott-Knott a 5\% de probabilidade de erro;

ns, ${ }^{*},{ }^{* *}$ não significativo, significativo a $5 \%$ e a $1 \%$ respectivamente.

$\mathrm{Na}$ tabela 2 está apresentado o desdobramento da interação dose $x$ época de avaliação para o atributo altura do perfilho primário. Ao 0 daa, a altura do perfilho primário variava de 22,67 a $28,37 \mathrm{~cm}$ nas plantas, não sendo observada diferença significativa entre os valores. Por outro lado, aos 25 daa essa altura variou de 23,83 a $46,17 \mathrm{~cm}$, havendo aumento significativo neste atributo, em relação à avaliação anterior, para as doses de 0 a 18 g e.a. ha ${ }^{-1}$, ou seja, não houve prejuízos no crescimento das plantas. Entre as doses de gliphosate, observouse aos 25 daa que 1,8 g e.a. ha ${ }^{-1}$ promoveu maior altura do perfilho primário entre todas as doses, valor que se diferenciou do tratamento testemunha. Por sua vez, os valores das alturas do perfilho primário para as doses de 3,6 e 7,2 g e.a. ha ${ }^{-1}$ não diferiram estatisticamente da testemunha, significando que não houve efeito hormótico da subdose; entretanto, também não houve qualquer efeito prejudicial a este atributo. A partir da dose de $18 \mathrm{~g}$ e.a. ha $^{-1}$ ocorreram significativas reduções na altura do perfilho. Esses resultados corroboram com os de VeLINI et al. (2008) ao observarem que doses subtóxicas de gliphosate (inferiores a $36 \mathrm{~g}$ e.a. ha $^{-1}$ ) estimularam o crescimento de várias espécies, tais como eucalipto, soja, milho e pinus e que essa estimulação ocorreu em diferentes partes da espécie avaliada. Do mesmo modo, CARBONARI et al. (2007b) constataram que a subdose de 3,6 g e.a. ha ${ }^{-1}$ estimulou o crescimento de eucalipto (Eucaliptus grandis), promovendo maior número de ramos laterais.

De acordo com Silva et al. (2007), o crescimento inicial rápido e uniforme da cultura permite atingir bom estande, possibilitando rápido fechamento de entrelinha com controle mais efetivo das plantas daninhas, além de cobertura do solo homogênea, o que leva a um eficiente aproveitamento da energia luminosa pela planta.

Quanto ao desdobramento da interação doses $x$ épocas de avaliação para número de folhas verdes, também não ocorreram diferenças significativas entre as médias ao 0 daa, quando os valores variaram de 7,66 a 14,00 folhas verdes por planta (Tabela 3). 
Tabela 2. Análise de variância do desdobramento da interação dose x época de avaliação, referente à altura dos perfilhos $(\mathrm{cm})$ aos 0 e 25 dias após a aplicação (daa) de gliphosate

\begin{tabular}{llc}
\hline \multirow{2}{*}{ Gliphosate } & \multicolumn{2}{c}{ Dias após a aplicação de gliphosate } \\
\cline { 2 - 3 } g e.a. ha ${ }^{-1}$ & 0 & 25 \\
0 & 25,43 a B \\
1,8 & 24,70 a B & 40,83 b A \\
3,6 & 22,67 a B & 46,17 a A \\
7,2 & 28,37 a B & 36,33 b A \\
18 & 25,27 a B & 39,83 b A \\
36 & 26,27 a A & 33,00 c A \\
72 & 23,90 a A & 27,83 d A \\
180 & 25,67 a A & 23,83 d A \\
360 & 24,57 a A & 26,67 d A \\
720 & 24,13 a A & 25,33 d A \\
\hline
\end{tabular}

Médias seguidas de letras diferentes, minúsculas na coluna e maiúsculas na linha, diferem entre si a $5 \%$ de probabilidade, pelo teste de Scott-Knott.

Tabela 3. Análise de variância do desdobramento da interação dose $x$ época de avaliação, referente a número de folhas verdes aos 0 e 25 dias após a aplicação de gliphosate

\begin{tabular}{cc}
\hline Gliphosate & \multicolumn{2}{c}{ Dias após a aplicação de gliphosate } \\
\cline { 2 - 3 } & 0
\end{tabular}

g e.a. ha ${ }^{-1}$

\begin{tabular}{lcc}
0 & 14,00 a A & 13,66 a A \\
1,8 & 13,00 a A & 12,00 a A \\
3,6 & 10,00 a A & 8,33 a A \\
7,2 & 11,67 a A & 7,33 a A \\
18 & 8,33 a A & 9,67 a A \\
36 & 11,33 a A & 8,67 a A \\
72 & 10,66 a A & 9,00 a A \\
180 & 9,33 a A & 4,67 b B \\
360 & 7,66 a A & 2,00 b B \\
720 & 11,33 a A & 0,67 b B \\
\hline
\end{tabular}

Médias seguidas de letras diferentes, minúsculas na coluna e maiúsculas na linha, diferem entre si a $5 \%$ de probabilidade, pelo teste de Scott-Knott.

Aos 25 daa, tanto entre doses quanto entre épocas de avaliação, observa-se forte redução no número de folhas verdes entre as doses de 180 a 720 g e.a. ha ${ }^{-1}$ A cor verde das folhas ocorre pela presença de pigmentos de clorofilas, responsáveis pela absorção de energia radiante para a realização da fotossíntese. Segundo YAMADA e CASTRO (2007), clorose é o sintoma mais comum e precoce de injúria do gliphosate às plantas, possivelmente devido à redução de clorofila, sendo similar à deficiência de ferro. NiLsson (1985) argumentou que há evidências de efeitos detrimentais causados pelo gliphosate sobre a síntese de ácido aminolevulínico (ALA), um precursor na biossíntese de clorofila. Meschede et al. (2007b) observaram redução significativa no conteúdo de clorofilas e carotenóides em cana-de-açúcar adulta sob efeito de gliphosate na dose comercial.

$\mathrm{O}$ índice SPAD foi aplicado devido à forte relação entre as leituras obtidas pelo clorofilômetro portátil e o conteúdo de clorofila (MARQUARD e TIPTON, 1987; MARKWELl et al., 1995). Na tabela 4 é apresentado o desdobramento da interação dose x época de avaliação para esse índice. No momento da aplicação das diferentes doses de gliphosate ( 0 daa) não havia diferença entre os valores, que variaram de 43,44 a 51,17. Quanto à avaliação aos 25 daa, houve decréscimo significativo dos valores em relação ao 0 daa, significando amarelecimento em todos os tratamentos. Por essa diminuição ter ocorrido também na testemunha, infere-se que possa ser atribuída ao fato das plantas já terem consumido grande parte do nitrogênio disponível no substrato. Na avaliação aos 25 daa observa-se redução significativa dos valores de índice SPAD a partir da dose de 7,2 g e.a. ha ${ }^{-1}$, exceto nas doses de 36 e 180 g e.a. ha ${ }^{-1}$. Segundo Meschede et al. (2007b), o gliphosate resulta na degradação da clorofila, causando amarelecimento das folhas. Schlemmer et al. (2005) observou que o uso do clorofilômetro SPAD-502 em plantas sob estresse hídrico inicial resultou em uma leitura subestimada do conteúdo de clorofila quando comparada ao conteúdo de clorofila obtido por extração, o que pode levar à indicação equivocada de que o estresse hídrico não afeta o conteúdo de clorofila no início.

Os resultados de massa fresca e seca da parte aérea e do sistema radicular são relacionados na tabela 5. A dose de $1,8 \mathrm{~g}$ e.a. ha ${ }^{-1}$ proporcionou aumento nas massas fresca e seca da parte aérea e na massa seca de raízes, diferenciando-se do tratamento testemunha. De uma maneira geral, doses inferiores a 7,2 g e.a. ha ${ }^{-1}$ não promoveram danos a esses atributos. Resultados semelhantes foram observados por CARBONARI et al. (2007b) ao concluírem que a aplicação de doses entre 3,6 e 7,2 g e.a. ha ${ }^{-1}$ estimulam o crescimento de eucalipto (Eucaliptus grandis), promovendo maior biomassa de parte aérea e raízes. Em trapoeraba (Commelina benghalensis), MESCHEDE et al. (2007a) verificaram efeitos positivos no crescimento e desenvolvimento da parte aérea e radicular com subdoses entre 2 e 24 g e.a. ha ${ }^{-1}$. 
Tabela 4. Análise de variância do desdobramento da interação dose $x$ época de avaliação, referente ao índice SPAD aos 0 e 25 dias após a aplicação de gliphosate

\begin{tabular}{llc}
\hline \multirow{2}{*}{ Gliphosate } & \multicolumn{2}{c}{ Dias após a aplicação de gliphosate } \\
\cline { 2 - 3 } g e.a. ha ${ }^{-1}$ & & 25 \\
0 & 48,70 a A & \\
1,8 & 48,96 a A & 41,07 a B \\
3,6 & 43,44 a A & 35,00 a B \\
7,2 & 45,53 a A & 32,47 a B \\
18 & 45,30 a A & 29,56 b B \\
36 & 46,10 a A & 30,16 b B \\
72 & 51,17 a A & 36,30 a B \\
180 & 50,60 a A & 26,40 b B \\
360 & 48,70 a A & 37,87 a B \\
720 & 47,93 a A & 26,90 b B \\
\hline
\end{tabular}

Médias seguidas de letras diferentes, minúsculas na coluna e maiúsculas na linha, diferem entre si a $5 \%$ de probabilidade, pelo teste de Scott-Knott.

Tabela 5. Médias e coeficientes de variação de massa fresca da parte aérea (MFA), massa fresca da raiz (MFR), massa seca da parte área (MSA) e massa seca da raiz (MSR)

\begin{tabular}{|c|c|c|c|c|}
\hline Gliphosate & MFA & MFR & MSA & MSR \\
\hline g e.a. ha ${ }^{-1}$ & \multicolumn{4}{|c|}{$\mathrm{g}$} \\
\hline 0 & $131,67 \mathrm{~b}$ & $56,67 \mathrm{a}$ & $18,61 \mathrm{~b}$ & $6,26 \mathrm{~b}$ \\
\hline 1,8 & $231,67 \mathrm{a}$ & $73,33 \mathrm{a}$ & $33,69 a$ & $9,01 \mathrm{a}$ \\
\hline 3,6 & 86,67 c & $65,00 \mathrm{a}$ & $16,78 \mathrm{~b}$ & $6,95 \mathrm{~b}$ \\
\hline 7,2 & $108,33 \mathrm{~b}$ & $58,33 \mathrm{a}$ & $18,92 \mathrm{~b}$ & $7,12 \mathrm{~b}$ \\
\hline 18 & $80,00 \mathrm{c}$ & $51,67 \mathrm{a}$ & $12,30 \mathrm{c}$ & $5,26 \mathrm{~b}$ \\
\hline 36 & $56,67 d$ & $50,00 \mathrm{a}$ & $12,08 \mathrm{c}$ & $5,23 \mathrm{~b}$ \\
\hline 72 & $41,67 \mathrm{~d}$ & $41,67 \mathrm{a}$ & 11,27 c & $4,16 \mathrm{c}$ \\
\hline 180 & $36,67 d$ & $11,67 \mathrm{~b}$ & $10,33 \mathrm{c}$ & $2,20 \mathrm{~d}$ \\
\hline 360 & $30,00 \mathrm{~d}$ & $15,00 \mathrm{~b}$ & $04,47 \mathrm{~d}$ & $1,90 \mathrm{~d}$ \\
\hline 720 & $15,00 \mathrm{~d}$ & $8,33 \mathrm{~b}$ & $4,21 \mathrm{~d}$ & $1,10 \mathrm{~d}$ \\
\hline CV (\%) & 21,02 & 26,75 & 22,71 & 22,88 \\
\hline Média Geral & 81,83 & 43,17 & 14,34 & 4,92 \\
\hline
\end{tabular}

Médias seguidas de letras diferentes diferem entre si a $5 \%$ de probabilidade, pelo teste de Scott-Knott.

Houve efeito estimulante da aplicação de gliphosate na subdose de $1,8 \mathrm{~g}$ e.a. ha ${ }^{-1}$ no desenvolvimento inicial da cana-de-açúcar, indicando potencial aplicação desse manejo para obtenção de melhores estandes após a brotação, a fim de conseguir melhor exploração do ambiente pela planta. Por meio dos resultados verifica-se que o desenvolvimento inicial da cana-de-açúcar pode ser positivamente responsivo à aplicação de subdoses de gliphosate. Assim, surge a perspectiva de que outros estudos devam ser realizados a fim de obter mais informações sobre o desenvolvimento da cultura da cana-deaçúcar em condições de cultivo em campo, em diferentes variedades, além do efeito sobre a produtividade.

\section{REFERÊNCIAS}

BLANCO, F.M.G. Controle das plantas daninhas na cultura da cana-de-açúcar. In: REUNIÃO ITINERANTE DO INSTITUTO BIOLÓGICO, 9, 2003, Catanduva, SP. Anais... Catanduva: Instituto Biológico, 2003. p.91-97.

CARBONARI, C.A.; MESCHEDE, D.K.; VELINI, E.D. Efeitos da aplicação de glyphosate no crescimento inicial de mudas de eucalipto submetidas a dois níveis de adubação fosfatada. In: SIMPÓSIO INTERNACIONAL SOBRE GLYPHOSATE, 1., 2007, Botucatu,SP. Anais... Botucatu: Faculdade de Ciências Agronômicas, UNESP, 2007a. v.1. 342p. p.68-70.

CARBONARI, C.A.; MESCHEDE, D.K.; VELINI, E.D.; GUERRINI, I.A. Acúmulo de fósforo em plantas de eucalipto de diferentes doses de glyphosate. In: SIMPÓSIO INTERNACIONAL SOBRE GLYPHOSATE, 1., 2007, Botucatu,SP. Anais... Botucatu: Faculdade de Ciências Agronômicas, UNESP, 2007b. v.1. 342p. p.76-78.

CASTRO, C. R. P.; VIEIRA, L. E. Aspectos gerais dos reguladores vegetais. In: CASTRO, C.R.P.; VIEIRA, L.E. Aplicações de reguladores vegetais na agricultura tropical. Guaíba: Agropecuária, 2001. Cap. 5, p.25-33.

FRANZ, J. E. Discovery, development and chemistry of glyphosate. In: GROSSBARBD, E.; ATKISON, D. The herbicide glyphosate. London: Butterworths, 1985. Cap. 1, p.3-17.

MACIEL, C.D.G.; VELINI, E.D.; SANTOS, R.F.; VIANA, A.G.P. Crescimento do curauá branco sob efeito de subdoses de glyphosate. In: SIMPÓSIO INTERNACIONAL SOBRE GLYPHOSATE, 1., 2007, Botucatu, SP. Anais... Botucatu: Faculdade de Ciências Agronômicas, UNESP, 2007. v.1. 342p. p.71-75.

MARKWELL, J.; OSTERMAN, J.C.; MITCHELL, J.L. Calibration of the Minolta SPAD-502 leaf chlorophyll meter. Photosynthesis Research, v.46, p.467-472, 1995.

MARQUARD, R.D.; TIPTON, J.L. Relationship between extractable chlorophyll and an in situ method to estimate leaf greenness. HortScience, v.22, p.1327, 1987.

MESCHEDE, D.K.; CARBONARI, C.A.; VELINI, E.D. Efeito de subdoses de glyphosate sobre o crescimento e desenvolvimento de Commelia benghalensis. In: SIMPÓSIO INTERNACIONAL SOBRE GLYPHOSATE, 1., 2007, Botucatu,SP. Anais... Botucatu: Faculdade de Ciências Agronômicas, UNESP, 2007a. v.1, p.65-67. 
MESCHEDE, D.K.; VELINI, E.D.; CARBONARI, C.A.;CORRÊA, M.R. Determinação do teor de lignina e celulose em amostras de Brachiaria decumbens submetidas á subdose de glyphosate. In: SIMPÓSIO INTERNACIONAL SOBRE GLYPHOSATE, 1., 2007, Botucatu,SP. Anais... Botucatu: Faculdade de Ciências Agronômicas, UNESP, 2007b. v.1, p.82-84.

NELSON, A.; RENNER, K.A.; HAMMERSCHMIDT, R. Effects of protoporphyrinogen oxidase inhibitors on soybean (Glycine max L.) response, Sclerotinia sclerotiorum disease development, and phytoalexin production by soybean. Weed Technology, v.16, p.353-359, 2002.

NETTO, J. M. Maturadores e reguladores vegetais na cultura da cana-de-açúcar. In: EGATO, S.V.;PINTO, A.S.; JENDIROBA, E; NÓBREGA, J. C. M. (Org.). Atualização em produção de cana-de-açúcar. Piracicaba: CP 2, 2006. p.307-318.

NILSSON, G. Interactions between glyphosate and metals essential for plant growth. In: GROSSBARD, E.: ATKISON, D. The herbicide glyphosate. London: Butterworths, 1985. Cap. 4, p.35-47.

PULVER, E. L; RIES, S. K. Action of simazine in increasing plant protein content. Weed Science, Ithaca, v.21, p.233-237, 1973.

RIES, S. K.; CHMIEL, H.; DILLEY D.R.; FILNER, P. Increase in nitrate reductase activity and protein content of plants treated with simazine. Proceedings of National Academy of Science USA, v.58, p.526-532, 1967.

SCHLEMMER, M.R.; FRANCIS D.D.; SHANAHAN, J.F.; SCHEPERS, J. S. Remotely measuring chlorophyll content in corn leaves with differing nitrogen levels and relative water content. Agronomy Journal, v.97, p.106-112, 2005.

SILVA, M. A.; CARLIN S.D.; CAPUTO M. M. Tipos de colheita e épocas de aplicação de glifosate na erradicação de soqueiras de cana-de-açúcar. Pesquisa Agropecuária Brasileira, v.41, p.4349, 2006.

SILVA, M.A.; GAVA, G.J.C.; CAPUTO, M.M.; PINCELLI, R.P.; JERONIMO, E.M.; CRUZ, J.C.S. Uso de reguladores de crescimento como potencializadores do perfilhamento e da produtividade em cana soca. Bragantia, v.66, p.545552,2007

SOUTHAM, C.M.; ERLICH, J. Effects of extracts of western red-cedar heartwood on certain wood-decaying fungi in culture. Phytopathology, v.33, p.517-524, 1943.

VELINI, E.D.; ALVES, E.; GODOY M.C; MESCHEDE, D.K.; SOUZA, R. T.; DUKE, S. O. Glyphosate applied at low doses can stimulate plant growth. Pest Management Science, v.4, p.489-96, 2008.

WIEDMAN, S. J.; APPLEBY, A.P. Plant growth stimulation by sublethal concentrations of herbicides. Weed Research, Oxford, v.12, p.65-74, 1972.

YAMADA, T.; CASTRO, P.R.C. Efeitos do glifosato nas plantas: implicações fisiológicas e agronômicas. International plant nutrition institute. Piracicaba: Informações Agronômicas, 2007. 24p. (Boletim Técnico 119) 